\title{
Intracerebral Hemorrhage: Large Disease Burden but Less Therapeutic Progress
}

\author{
Dong-Wha Kang \\ Department of Neurology, Asan Medical Center, University of Ulsan College of Medicine, Seoul, Korea
}

The current issue of Journal of Stroke publishes three review articles regarding intracerebral hemorrhage $(\mathrm{ICH})$. ICH is caused by diverse etiologies including hypertension, cerebral amyloid angiopathy, trauma, vascular malformation, and drugs. Compared to ischemic strokes, the incidence of ICH is lower. However, the 2010 Global Burden of Disease (GBD) Study showed that the absolute number of hemorrhagic strokes increased $47 \%$ worldwide for the last two decades, ${ }^{1}$ mainly due to increased ICH in lowand middle-income countries. Moreover, as the population is aging, prevalence of $\mathrm{ICH}$ associated with amyloid angiopathy and anticoagulation is also increasing. Warfarin-associated ICH is associated with high mortality and disability. Currently, new oral anticoagulants (NOACs) rapidly replace vitamin $\mathrm{K}$ antagonists for stroke prevention in patients with atrial fibrillation; however, only one NOAC, dabigatran, has an antidote available. Management of NOAC-related ICH has become an emerging issue.

Blood pressure (BP) is usually elevated in the acute phase of ICH. Studies have been performed to determine whether intensive BP lowering is more beneficial than standard BP treatment in the early phase of ICH. The INTERACT-2 study showed that early intensive BP lowering (target systolic BP $<140 \mathrm{mmHg}$ ) was moderately associated with lower mortality or major disability rate than standard BP lowering (target systolic BP $<180 \mathrm{mmHg}$ ). ${ }^{2}$ However, the reasons for improved outcomes are unclear because changes in hematoma growth did not differ between the two groups. The ATACH-2 trial, which randomly assigned patients to the same BP targets as in INTERACT-2, showed that the outcomes were not different between the intensive and standard treatment groups. ${ }^{3}$ The hypothesis of these trials was based on the idea that intensive BP control might inhibit rebleeding or hematoma growth. However, the lower is not always the better. A substudy of INTERACT-2 showed a J-curve for BP-outcome interaction; systolic BP $<130 \mathrm{mmHg}$ as well as $>140 \mathrm{mmHg}$ after $\mathrm{ICH}$ was associated with poor outcomes. ${ }^{4}$ It is controversial whether the early rise in $\mathrm{BP}$ after $\mathrm{ICH}$ is an adaptive response to perfuse the perihematomal penumbral area or a simple deleterious event resulting in rebleeding or expansion of edema. Future trials should consider cerebral hemodynamics in individual patients to allow tailored BP management strategies.

Finally, there is limited evidence regarding the benefits of surgical treatment of ICH. The STICH trials could not demonstrate the overall benefit of early surgical interventions on mortality and functional outcomes in patients with ICH., ${ }^{5,6}$ However, the results do not negate the potential benefit of early surgery in selected patients. In the subgroup analysis of STICH $I_{1}^{6}$ patients in the poor prognosis group (i.e., low Glasgow coma score and large hematoma volume) benefitted from early surgery, whereas those in the good prognosis group did not. Identification of patients who may benefit from early surgery on the basis of clinical, imaging, hematoma, and hemodynamic characteristics should be of great importance. Technical advances in surgical evacuation should also be established. Future trials in such selected patients with advanced surgical techniques are warranted.

Recently, there has been remarkable progress in endovascular therapy in hyperacute ischemic stroke. We are eagerly anticipating such major milestones in ICH therapeutics.

\section{References}

1. Krishnamurthi RV, Moran $A E$, Forouzanfar $M H$, Bennett DA, Mensah GA, Lawes CM, et al. The global burden of hemorrhagic stroke: a summary of findings from the GBD 2010 
study. Glob Heart 2014;9:101-106.

2. Anderson CS, Heeley E, Huang Y, Wang J, Stapf C, Delcourt C, et al. Rapid blood-pressure lowering in patients with acute intracerebral hemorrhage. N Eng/ J Med 2013;368:2355-2365.

3. Qureshi Al, Palesch YY, Barsan WG, Hanley DF, Hsu CY, Martin $\mathrm{RL}$, et al. Intensive blood-pressure lowering in patients with acute cerebral hemorrhage. N Engl J Med 2016;375:10331043.

4. Arima $H$, Heeley E, Delcourt $C$, Hirakawa $Y$, Wang $X$, Woodward $M$, et al. Optimal achieved blood pressure in acute intracerebral hemorrhage: INTERACT2. Neurology 2015;84:464-471.

5. Mendelow AD, Gregson BA, Fernandes HM, Murray GD, Teasdale GM, Hope DT, et al. Early surgery versus initial conservative treatment in patients with spontaneous supratentorial intracerebral haematomas in the International Surgical Trial in
Intracerebral Haemorrhage (STICH): a randomised trial. Lancet 2005;365:387-397

6. Mendelow AD, Gregson BA, Rowan EN, Murray GD, Gholkar A, Mitchell PM, et al. Early surgery versus initial conservative treatment in patients with spontaneous supratentorial lobar intracerebral haematomas (STICH II): a randomised trial. Lancet 2013;382:397-408.

Correspondence: Dong-Wha Kang

Department of Neurology, Asan Medical Center, University of Ulsan College of Medicine, 88 Olympic-ro 43-gil, Songpa-gu, Seoul 05505, Korea Tel: +82-2-3010-3440, Fax: +82-2-474-4691

Email: dwkang@amc.seoul.kr

The author has no financial conflicts of interest. 